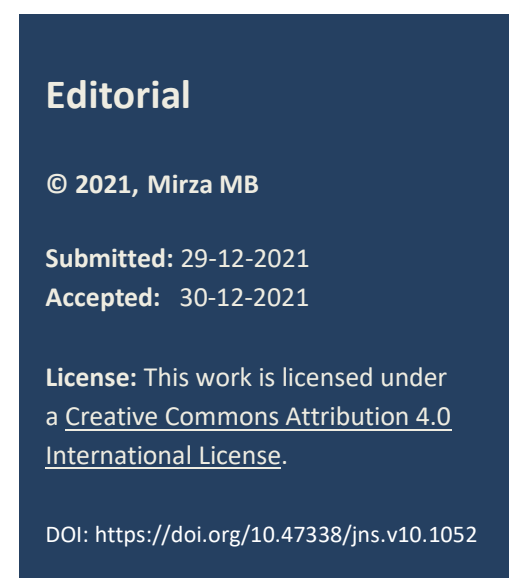

\section{The year 2021- Good for the surgical neonates in Pakistan}

\section{Muhammad Bilal Mirza*}

Editor in Chief, Journal of Neonatal Surgery.

Correspondence*: Dr. M. Bilal Mirza, MBBS, MS, CHPE, Associate Professor of Pediatric Surgery, University of Child Health Sciences, Lahore. E-mail: blmirza@gmail.com
I am quite elated to elaborate exciting developments for surgical neonates in Pakistan, happened this year. We now believe that the outcomes of the surgical neonates cannot be improved, merely based on the research, and the improvement in the clinical practice through quality improvement projects (QIP), is equally important.

Clinical audit-a quality improvement tool- was introduced at the University of Child Health Sciences
Lahore, by Dr. Irfan Waheed Khawaja (Professor of Neonatology) in 2013. Few workshops were conducted at various other hospitals but could not get due attention at that time. With continued urge, now the clinicians are turning to it considering it a potential tool for the improvement of the clinical practice. Dr. Khawaja recently introduced a local made costeffective neonatal transport system for improving the practice of neonatal transfer (Fig. 1).

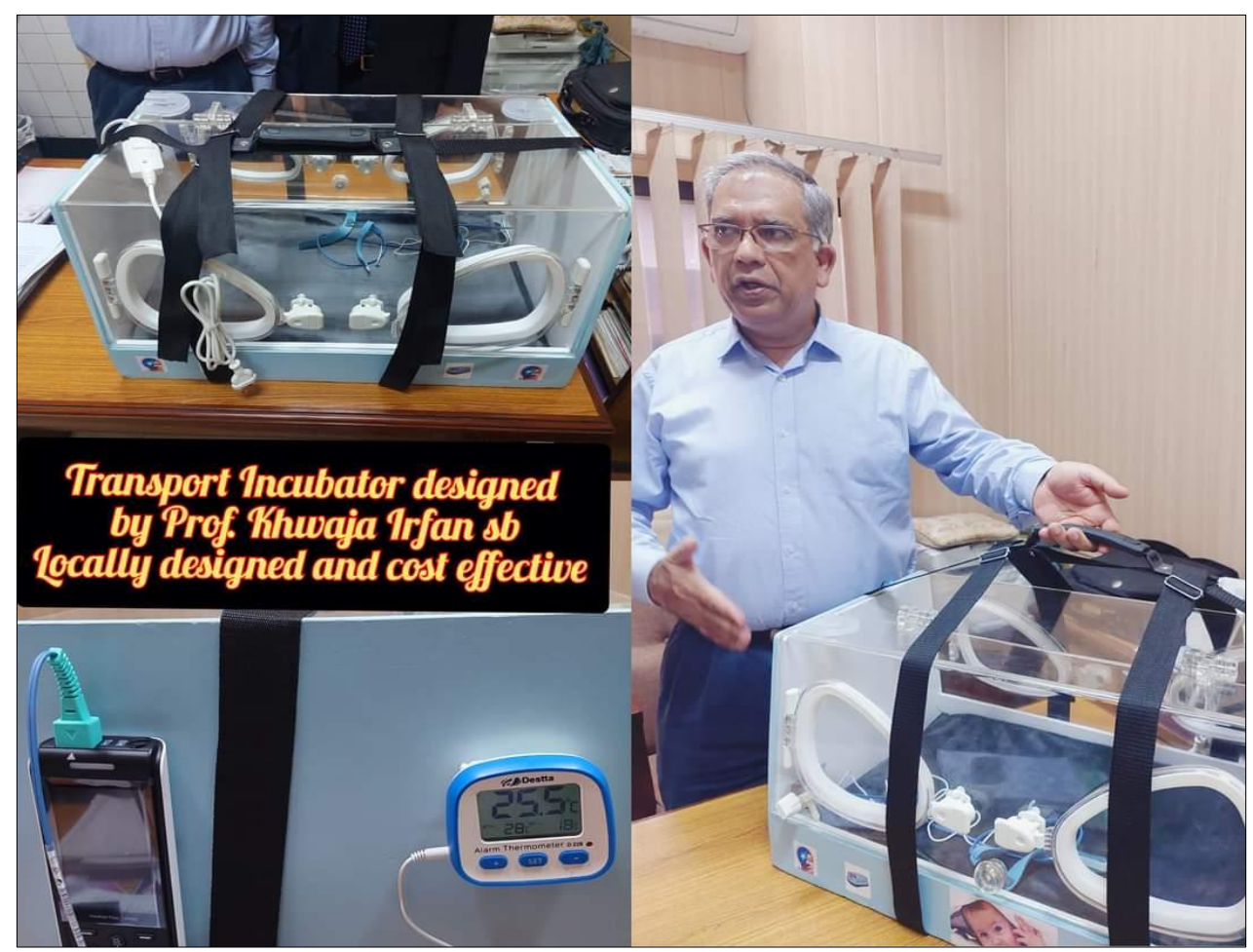

Figure 1: Prof. Irfan Waheed Khawaja introducing a locally made neonatal transport system with in-built temperature monitoring and management system, and pulse oximeter.

Although QIP is not a research project and cannot be generalized, improvement in individual neonatal setups can collectively improve the overall Neonatal Mortality Rate which is currently quite high in Pakistan. Categorizing the prognostic factors into the patient- related factors (late presentation, prematurity, low birth weight, development of sepsis), managementrelated factors (surgical technique, expertise, perioperative care), and system-related factors (availability of dedicated neonatal surgical ICU, operation rooms, 
and modern gadgets of monitoring), the patientrelated factors are difficult to modify however the management-related factors and to some extent, the system-related factors can be improved with QIP. The improvement in the clinical practice will ultimately translate into improved clinical outcomes.

Two exciting events that happened in the year will pave the way to improve health care. The first international conference on "Quality Improvement" was arranged by Prof. Irfan Waheed Khawaja and Prof. Khadija (Endocrinologist of Services Institute of Medical Sciences Lahore) in collaboration with the National Institute of Health Research, UK (NIHR) in November 2021. This event was well attended and participants across the country and abroad presented their quality improvement projects including clinical audits and PDSA cycles. Health authorities also promised to include clinical audits in the residency program.

The second event was a symposium on "Neonate as a Surgical Patient" arranged by Dr. Mudassar Gondal (Pediatric surgeon at Rawal Medical University) in collaboration with the Association of Paediatric Surgeons of Pakistan (APSP) (Fig. 2). It was well attended by pediatric surgeons from every part of the country. It sensitized the pediatric surgeons about special attention, and care needed by the surgical neonates. Various aspects of neonatal surgical care including the development of the modern infrastructure and training of the nursing staff were discussed and planned. Idea of Neonatal surgery as a separate specialty also aired in the symposium.

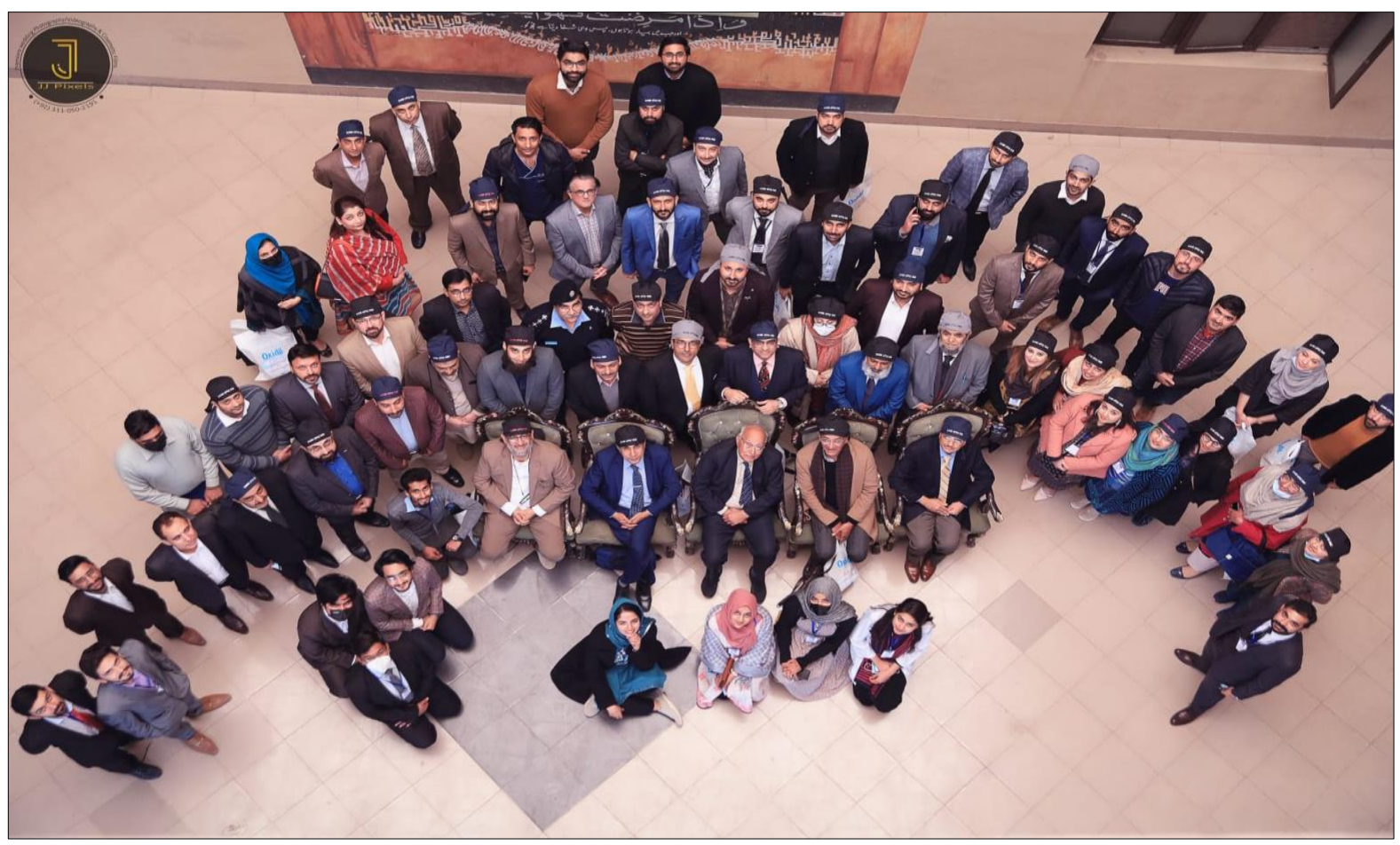

Figure 2: Participants of the symposium on Neonate as a Surgical Patient.

Considering the importance of QIP in improving clinical practice, the editorial team of the Journal of Neonatal Surgery is pleased to start a section on QIP. We will publish the quality improvement projects including but not limited to Clinical audits and PDSA cycles in this category. I hope this will be another step in consolidating the aims and objectives of this journal. With this, I on the behalf of the editorial team wish our readers, authors, reviewers, and well-wishers, a Happy and Prosperous New Year
Acknowledgements: $\mathrm{Nil}$

Conflict of Interest: Author is editor in chief of the journal.

Source of Support: Nil

Consent to Publication: No clinical picture is used in the manuscript.

Author Contributions: Author(s) declared to fulfil authorship criteria as devised by ICMJE and approved the final version. 